The Open Civil Engineering Journal
CrossMark
Content list available at: www.benthamopen.com/TOCIEJ/
DOI $10.2174 / 1874149501610010738$

RESEARCH ARTICLE

\title{
The Method of Model Approximation for the Analysis of Semi-rigid Connection with Parameter Correlation
}

\author{
Xian Dong ${ }^{1}$ and Zhan Wang ${ }^{1,2, *}$ \\ ${ }^{\text {I} D e p a r t m e n t ~ o f ~ C i v i l ~ E n g i n e e r i n g, ~ S o u t h ~ C h i n a ~ U n i v e r s i t y ~ o f ~ T e c h n o l o g y, ~ G u a n g z h o u ~ 510640, ~ C h i n a ~}$ \\ ${ }^{2}$ State Key Laboratory of Subtropical Building Science, South China University of Technology, Guangzhou 510640, \\ China
}

\begin{abstract}
The moment-rotation model is the main study of semi-rigid connection, moreover, the sensitivity coefficients of parameters on semi-rigid connection are the precondition of a moment-rotation model. There are two issues in the current research: the defective models would be obtained without considering the correlation among the parameters of the structure; for the higher precision sensitivity coefficients, a large number of finite element simulations would be conducted and this process leads to intensive computation. According to these problems, the sampling of the parameters with considering the correlation and the approximate model which was constructed by numbered finite element simulations and improved chaotic particle swarm algorithm are proposed in this paper. Two mathematical example are conducted and the approximate models represent a high degree of accuracy. A new sensitivity calculation is proposed based on the correlation parameters and the approximate model, the moment-rotation model of extended end plate connection obtained from this algorithm has the properties of high computational efficiency and explicit physical meaning.
\end{abstract}

Keywords: Approximate model, Correlation between parameters, Moment-rotation model, Semi-rigid connection, Sensitivity analysis.

\section{INTRODUCTION}

Beam-column connections, as an important part of the steel frame structure, have been divided into three categories according to the characteristic of force deformation: rigid connection, pinned connection, and semi-rigid connection. In the design of steel frame structure, engineers design the beam-column connection as so called rigid joint or pinned connection to resist the lateral and reduce the lateral displacement efficiently of structures usually, but both types are the artificial assumptions, in practical engineering, all of the connections are the semi-rigid connections. The investigation on the behavior of semi-rigid connection began in 1917 [1], moreover, the moment-rotation curve takes on a more important role such as linear models [2], polynomial models and exponential models [3].

The plastic hinge method has been widely used in nonlinear analysis of semi-rigid steel frame. Liew and Kim $[4,5]$ presented the refined plastic hinge analysis model for plane frame, Chan [6] proposed springs-in-series model with material nonlinearity at each end of the element for semi-rigid connections. Hu shu-jun [7] presented an element with springs-in-series at each end for semi-rigid steel frame with elements of internal plastic hinge, it could consider the effect of semi-rigid connections and material nonlinearity. A Saritas et al. [8] presented a mixed formulation frame finite element with internal semi-rigid connections for the nonlinear analysis of steel structures. The formulation of the element also considers the presence of linear or nonlinear behavior of semi-rigid connection behavior without additional

\footnotetext{
* Address correspondence to this author at the Department of Civil Engineering, South China University of Technology, Guangzhou 510640, China; Laboratory of Subtropical Building Science, South China University of Technology, Guangzhou 510640, China; Tel/Fax: 13560040432; E-mail: wangzhan@scut.edu.cn
} 
nodes and degrees of freedom.

While the experimental and theoretical modeling of semi-rigid connections have been performed by researches, they are almost based on the assumption that the variables of structures are completely determined, which means evaluating the effects of variables on the structures by varying input parameters one at a time then holding other parameters at determined values with ignoring the relationship among these variables [9]. The structure contains many parameters in the analysis of semi-rigid connections, there may be correlations between each parameter. These correlations impacted largly on the final output responses of the structures. The wrong sensitivity coefficients would be calculated by analyzing the effect of parametric variables on structures without considering the relativity of parameters [10].

There are a few researches on the parameters correlation [11], and more importantly, the moment-rotation curves regressed by this assumption would be counterintuitive in practical applications, so it is worth to study the semi-rigid connection based on the correlations between the parameters. The analysis of the sensitivity coefficients is to evaluate the variations of the structural response due to the variations of the design parameters [12], it is the precondition to study the moment-rotation model. The probability design model of finite element analysis and the basic theory Monte Carlo are implemented as the basic means to obtain the sensitivity coefficients. By use of Monte Carlo, a lot of computer time and software would be taken into account in finite element simulation. However, despite growing computing power, the software defects still represent a significant weakness.

Herein a different way in the study of the moment-rotation model is proposed. This method includes the sampling of correlation parameters based on the theory of Monte Carlo and the approximate model that aims to not dependent on the finite element analysis software. The approximate model constitutes a fusion between the finite element simulation analysis and hybrid neural network which has been optimized by the method of improved chaotic particle swarm. Two mathematical examples have been used to illustrate the applicability and accuracy of the approximate model. For representation of the moment-rotation model of semi-rigid connection, an extend end plate connection is implemented while considering the correlations and sensitivity of parameters in the literature. The proposed method is developed for the analysis of sensitivity and the moment-rotation model with efficiently.

\section{EXPERIMENT AND FINITE ELEMENT ANALYSIS OF EXTENDED END PLATE CONNECTION}

\subsection{Analysis of Experiment}

This experimental program was an extend end plate connection which was subjected to static loads. This test specimen was bolted to the column flange and the plate and M20 high-strength bolt was employed, the column section was HE340 and the beam was IPE300. The geometry and arrangement of the instrumentation of the joint are shown in Fig. (1) [13]. Based on the available test data, detailed geometry of the specimen are shown in Table 2, the average characteristics of the connection are set out in Table $\mathbf{1}$, where $E$ is the elastic modulus, $E_{\mathrm{st}}$ is the strain hardening modulus, $f_{y}$ is yield strength, $f_{u}$ is the tensile stress, $\varepsilon_{e}$ is the elastic strain, $\varepsilon_{s t}$ is the strain of the initial hardening point, $\varepsilon_{u n i}$ is the uniform strain, $\varepsilon_{u}$ is the ultimate strain.

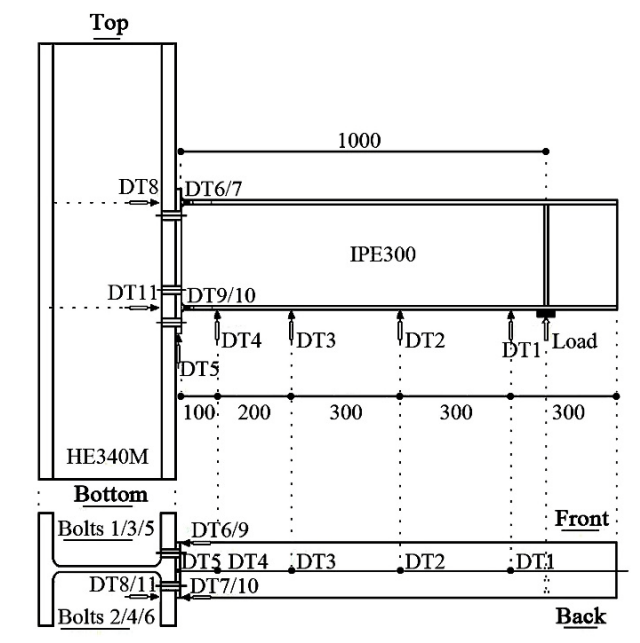

Fig. (1). Geometry of the specimen [13]. 
Table 1. Characteristic values of the structural steels [13].

\begin{tabular}{|c|c|c|c|c|c|c|c|}
\hline Components & $\boldsymbol{E} / \mathbf{M P a}$ & $\boldsymbol{E}_{\mathbf{s t}} / \mathbf{M P a}$ & $\boldsymbol{f}_{\mathbf{y}} / \mathbf{M P a}$ & $\boldsymbol{f}_{\mathbf{u}} / \mathbf{M P a}$ & $\boldsymbol{E}_{\boldsymbol{s t}}$ & $\boldsymbol{\varepsilon}_{u n i}$ & $\boldsymbol{\varepsilon}_{\boldsymbol{u}}$ \\
\hline Plate and column & 209856 & 2264 & 340.12 & 480.12 & 0.015 & 0.224 & 0.361 \\
\hline Beam web & 208332 & 1856 & 299.12 & 446.25 & 0.016 & 0.235 & 0.464 \\
\cline { 2 - 8 } Beam flange & 209496 & 1933 & 316.24 & 462.28 & 0.016 & 0.235 & 0.299 \\
\hline Bolt & 223166 & - & 857.33 & 913.78 & - & - & 0.272 \\
\hline
\end{tabular}

Table 2. Actual geometry of the connection [13].

\begin{tabular}{|c|c|c|c|c|c|c|c|c|c|c|c|}
\hline \multicolumn{9}{|c|}{ Column/mm } & \multicolumn{5}{c|}{ Beam/mm } \\
\hline$h$ & $b$ & $t_{\mathrm{f}}$ & $t_{\mathrm{w}}$ & $H_{\text {up }}$ & $H_{\text {low }}$ & $h$ & $b$ & $t_{\mathrm{f}}$ & $t_{\mathrm{w}}$ & $L_{\text {beam }}$ & $L_{\text {load }}$ \\
\hline 376.0 & 307.5 & 40.21 & 21.0 & 175.0 & 219.0 & 300.45 & 150.5 & 10.76 & 7.2 & 1200 & 1002.5 \\
\hline
\end{tabular}

\subsection{Finite Element Analysis}

This beam column joint is modeling by Ansys 14, according to the symmetry, the finite element model is the $1 / 2$ structure of the solid model, the types of elements including SOLID95, TARGE170, CONTA174 and PRETS179. SOLID95 is a higher-order version of the 3-D 8-node solid element SOLID45. It can tolerate irregular shapes without as much loss of accuracy. Each component of the extended end plate connection was modeled by solid element SOLID95, all the interfaces of the contact including the interfaces between the extended end plate and the column flange, the bolt and the end plate. The bolt and column flange were modeled by TARGE170 and CONTA174 elements. The pretension force of bolts was modeled by PRETS179 elements. The finite element model consists of 9043 SOLID95, 625 CONTA174, 130 TARGE170 and 234 PRETS179. The analysis of semi-rigid joints is finite element static, the loading process is divided into two steps: first, the pre tension is applied at the high strength bolts, and then concentrated force at the beam end.

The finite element model is shown in Fig. (2), the stress-strain curve of steel beam and end plate is shown in Fig. (3). The displacement of DT2 as show in Fig. (1) which was to calculate the rotation of the structure and the expressions are represented as follows [14]:

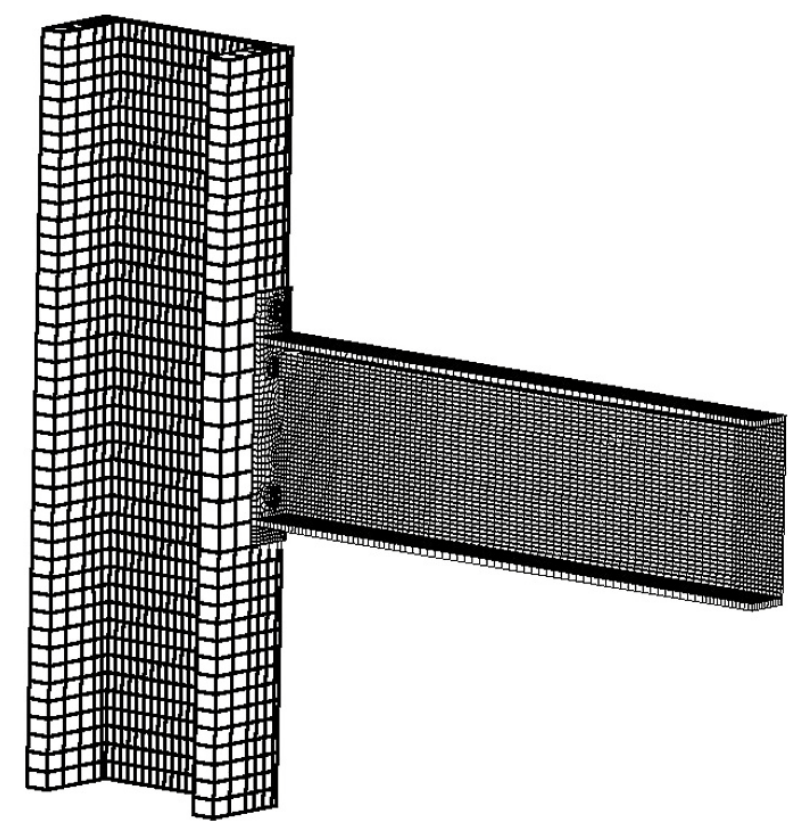

Fig. (2). Finite element model. 


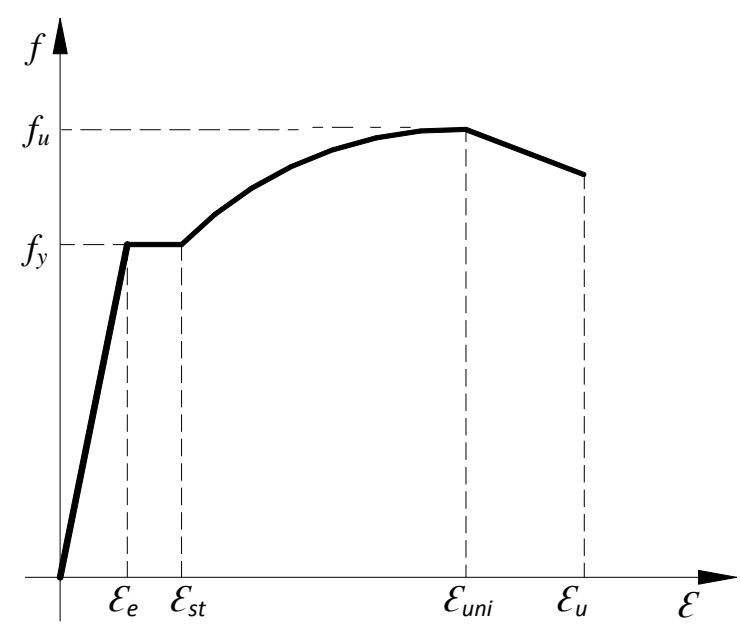

Fig. (3). Stress-strain curves of steel [13].

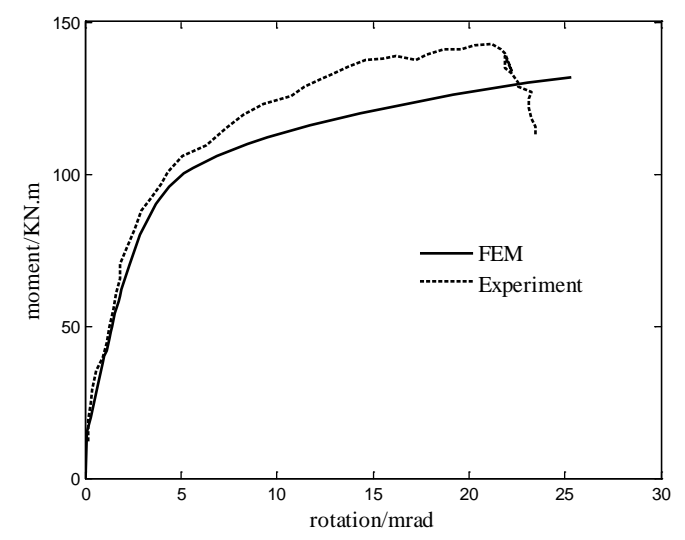

Fig. (4). Moment-rotation curve of DT2.

$$
\begin{aligned}
& \theta_{r}=\arctan \left(\frac{\delta_{D T 2}-\delta_{D T 5}-\delta_{b, e 1(D T 2)}}{600}\right) \\
& \delta_{b, e l(D T 2)}=-\frac{P}{E_{b} I_{b}}\left(\frac{x_{D T 2}^{3}}{6}-\frac{L_{l o a d} x_{D T 2}^{2}}{2}\right)
\end{aligned}
$$

Where $\delta_{D t 2}$ is the vertical displacement of DT2 and $\delta_{D t 5}$ is the vertical displacement of DT5, $\delta_{b, e l(D t 2)}$ is the beam elastic deflection at linear variable displacement transducer of DT2 evaluated by equation(2). Where $E_{b}$ is the beam elastic modulus, $I_{b}$ is the moment of inertia of beam, $L_{\text {load }}$ is the distance between the load application point and the face of the end plate, $P$ is the load at the free end of beam and $x_{D T 2}$ is the distance of DT2 which are shown in Fig. (1). The comparison between experimental results and finite element model is shown in Fig. (4), where the phantom is simulated by finite element analysis, the solid line is the experimental value (numerical value in reference [13]). Agreement appears much closer between the two lines, the finite element model can be used later in the analysis.

\section{MONTE CARLO SAMPLING WITH THE CORRELATION OF THE PARAMETERS}

The purpose of Monte Carlo sampling is to meet the requirement of accuracy in probability, but random sampling may leads to the paradoxical conclusions. It is noted that Monte Carlo sampling with correlation parameters is in accordance with the parameters changing. 


\subsection{Transformation Between Correlated Non Normal Variables and Correlated Normal Variables}

A mathematical theorem is that the normal variables are independent when the correlation coefficients are zero. Non-normal random variables will be converted into normal random variables by the functions with equal probability transformation. A random vector $X=\left[X_{1} X_{2} \ldots X_{3}\right]$ and a standard normal distribution vector $Y=\left[Y_{1} Y_{2} \ldots Y_{3}\right]$ are used in this transformation. Then the relationship between random distribution and normal distribution are given by:

$$
\left\{\begin{array}{c}
F_{i}\left(x_{i}\right)=\Phi\left(y_{i}\right) \\
x_{i}=F_{i}^{-1}\left[\Phi\left(y_{i}\right)\right]
\end{array} \quad i=1,2 \cdots n\right.
$$

Joint probability density functions deriving from the rules of implicit function derivation are expressed as follows:

$$
\begin{aligned}
& f_{x}(x)=\frac{f_{1}(x) f_{2}(x) \cdots f_{n}(x)}{\varphi\left(y_{1}\right) \varphi\left(y_{2}\right) \cdots \varphi\left(y_{n}\right)} \varphi_{n}\left(y, \rho_{0}\right) \\
& \varphi_{n}\left(y, \rho_{0}\right)=\frac{1}{\sqrt{(2 \pi)^{n} \operatorname{det}\left(\rho_{0}\right)}} \exp \left(-\frac{1}{2} y^{T} \rho_{0}^{-1} y\right)
\end{aligned}
$$

where $F_{i}(\bullet)$ is a marginal probability distribution function, $F_{i}^{-1}[\bullet]$ is the inverse function of $F i(\bullet), \Phi(\bullet)$ is a standard normal distribution function, $\rho$ is the equivalent correlation coefficient matrix of $Y, f_{i}(\bullet), i=1,2, \ldots n$ is the marginal probability density function corresponding with $F_{i}(\bullet), \varphi(\bullet)$ is the probability density function of standard normal variables, $\operatorname{det}(\bullet)$ represents the matrix determinant, $(\bullet)^{-1}$ is the function of matrix inversion. The correlation coefficient $\rho_{0 x y}$ of standard normal random vector $y$ can be derived from equation (6) for the marginal probability density function of random variables and $\rho$ have been calculated.

$$
\begin{aligned}
& \rho_{i j}=\int_{-\infty}^{+\infty} \int_{-\infty}^{+\infty}\left(\frac{x_{i}-\mu_{x_{i}}}{\sigma_{x_{i}}}\right)\left(\frac{x_{j}-\mu_{x_{j}}}{\sigma_{x_{j}}}\right) f_{X_{i} X_{j}}\left(x_{i}, x_{j}\right) d x_{i} d x_{j} \\
& =\int_{-\infty}^{+\infty} \int_{-\infty}^{+\infty}\left(\frac{F_{i}^{-1}\left(\Phi\left(y_{i}\right)\right)-\mu_{x_{i}}}{\sigma_{x_{i}}}\right)\left(\frac{F_{j}^{-1}\left(\Phi\left(y_{j}\right)\right)-\mu_{x_{j}}}{\sigma_{x_{j}}}\right) \bullet \phi_{2}\left(y_{i}, y_{j}, \rho_{0 i j}\right) d y_{i} d y_{j}
\end{aligned}
$$

Where $\rho_{i j}$ is the correlation coefficient between $x_{i}$ and $x_{j}$ of random vector $X, \rho_{0 i j}$ is the correlation coefficient matrix component of standard normal random vector $y, f_{X X}\left(x_{i}, x_{j}\right)$ is the joint probability density function about the ith and $j t h$ variables.

\subsection{Transformation Between Correlated Normal Variables and Independent Normal Variables}

Suppose that the correlation coefficient matrix $\rho$ have been calculated by the section 3.1, an intermediate variable $M$ is represented as

$$
M=V \cdot \sqrt{\Lambda}
$$

Where $\Lambda$ is a diagonal matrix composed by the eigenvalues $\lambda$ belonging to the correlation coefficient matrix $\rho_{0}, V$ is a matrix composed by the eigenvectors $v$ corresponding with the eigenvalues $\lambda$. The relationship between independent standard normal variables $U$ and correlated standard normal variables $Y$ can be represented as follows:

$$
U=M^{-1} \bullet Y
$$

The Monte Carlo sampling process considering the correlation between parameters is listed as below:

1. Generate random variables $X$ with n-dimension following normal distribution, assumed a computational accuracy $\theta$; 
2. Calculate the correlation coefficient matrix of normal distribution random variables;

3. Transform correlated random normal variables to random independent variables by the algorithm of section 3.1 ;

4. Calculate the correlation coefficient matrix $\rho$ of the original model, obtain the equivalent correlation coefficient matrix $\rho$ according to the literature [15];

5. Generate random variables following normal distribution with the correlation coefficient $\rho_{0}$ according to the inverse transformation of section 3.1, get the absolute values of the differences between correlation coefficient matrix of the generated variables and $\rho_{0}$, if all the differences are less than $\theta$, go to step 6 , otherwise, go back to step 2;

6. Transform the correlated random normal variables with correlation coefficient matrix $\rho_{0}$ to the correlated random non-normal variables based on the equal probability transformation.

The beam height and width, thickness of web and thickness of flange which are belong to IPE steel are select as the statistical parameters. According to the partial correlation analysis by SPSS [16], we can get the conclusion that there are the correlations between height and width, thickness of flange and thickness of web, the correlation coefficients are 0.87 and 0.9 respectively.

\section{THE METHOD OF APPROXIMATE MODEL}

\subsection{The Algorithm of Neural Network with Improved Chaos Particle Swarm}

The approximate model is constructed by the feedback neural network which has been optimized by chaos particle swarm. Feedback networks is a nonlinear dynamic system with significant learning capacity by small information, its state is changing continuously until they reach an equilibrium point. Also, they remain at the equilibrium point until the input changes and a new equilibrium would to be found. Its algorithm uses gradient descent search technique to minimize a cost function equal to the mean square difference between the desired and the actual outputs [17]. In 1989, Hornik et al. have proved that any complexity function could be approximated by a feedforward neural network with a single hidden layer [18, 19]. Yin zhi-guang et al. [20] presented a BP neural network model of coal seam gas permeability. It was built based on the coal mechanical properties, the accuracy of the model was tested and the BP neural network model match the measured data well. Han hong-gui et al. [21] presented a new flexible RBF neural network to the problem of the structure design.

The principle of feedback neural network is expressed as follows:

$$
\begin{cases}x_{k}^{\prime}=f\left(\sum_{i=0}^{n-1} w_{i k} x_{i}-b_{k}\right), & k=0,1,2, \cdots, n_{1}-1 \\ y_{l}=f\left(\sum_{k=0}^{n_{1}-1} w_{k l}^{\prime} x_{k}^{\prime}-b_{l}^{\prime}\right), & l=0,1,2, \cdots, m-1\end{cases}
$$

Where $y_{l}$ is the network output, $x_{i}$ is the network input, $x^{\prime}{ }_{k}$ is hidden layer neuron, $w_{i k}$ is the weight of input layers to hidden layers, $b_{k}$ is the threshold value of input layers to hidden layers, $w_{k l}$ ' is the weight of hidden layers to output layers, $b_{l}{ }^{\prime}$ is the threshold value of hidden layers to output layers, $f$ is a transfer function.

For the complex optimization problems, the phenomenon of slow convergence and falling into the local extreme is increasingly outstanding. The neural network whose weights and threshold value are optimized by the combination of chaotic and particle swarm optimization algorithm would jump out of local minima, the particles also may keep the diversity of population and improve global optimization performance [22]. This algorithm has been improved by optimizing the particles alternately between steady and chaos [23]. Fitness functions according to the objective function would be established to estimate whether the particles were in premature convergence or not. In this paper, the relative errors between the network outputs and the actual response are employed as the fitness functions. To insure the functions are nonnegative, the expressions are represented as Eq. (10), where $u(x)$ is the judgment function of premature convergence.

$$
u(x)=\sqrt{\sum_{i=1}^{n}\left(y_{i}-f\left(x_{i}\right)\right)^{2}} / \sum_{i=1}^{n} f\left(x_{i}\right)
$$


The determination about whether the status of particle swarms is in steady or chaos can be given as Eq. (11), abs indicates the absolute value of the movement distance of the current particle.

$$
\text { smove }=\operatorname{abs}\left(x_{i}(t)-x_{i}(t-1)\right)
$$

Where $t$ is a number of the particle evolutionary generations. It is considered that the particle has reached to the local optimization when smove or $u(x)$ is less than a limit value. The variables will get into chaos by chaotic searching. Logistic model can be given as:

$$
x_{n+1}=\mu-x_{n}^{2}, \quad n=1,2, \ldots
$$

Where $\mu$ is a control parameter deciding the state of the system, the system is totally in chaotic state when $\mu=2$.The procedures of chaotification are as follows:

(1) Make $x_{i, v}$ mapped into the interval $[-2.0,2.0]$ by Eq. (13), where $x_{i, v}$ is the location of particle $x_{i}$ in the $v t h$ dimension, $\left[a_{v}, b_{v}\right]$ is the domain of $x_{i, v}$ in the $v$ th dimension.

$$
h x_{i, v}=\frac{4 x_{i, v}-2\left(b_{v}+a_{v}\right)}{b_{v}-a_{v}}
$$

(2) Generate a chaotic sequence $h x_{i, v}^{1} h x_{i, v}^{2}, \ldots h x_{i, v}^{J}$ by iterating the equation $h x_{i}^{j+1}=2-h x_{i}^{j}$ for $j$ times;

(3) Map particles of the chaotic sequence to the primary searching space following the function $x_{i, v}^{j}=1 / 4\left\{2 b_{v}+2 a_{v}+\right.$ $\left.h x_{i, v}^{j}\left(b_{v}-a_{v}\right)\right\}$, chaos column points $x_{i}^{j}=\left(h x_{i, 1}^{j}, h x_{i, 2}^{j}, \ldots h x_{i, n}^{j}\right)$ in each dimensional chaotic sequence can be derived from Tent map [24]. In the particle swarm optimization of this paper, the particles would be divided into two kinds, the first kind particles are the weights from input layers to the hidden layers and hidden layers to the output layers, the second kind are the threshold values form input layers to hidden layers and hidden layers to the output layers. These matrixes are expressed as:

$\operatorname{Particle}(1)=\left[W_{1}, W_{2}\right]$

$$
\begin{aligned}
& W_{1}=\left[\begin{array}{cccc}
w_{1,1} & w_{1,2} & \cdots & w_{1, n} \\
w_{2,1} & w_{2,2} & \cdots & w_{2, n} \\
\vdots & \vdots & \vdots & \vdots \\
w_{m, 1} & w_{m, 2} & \cdots & w_{m, n}
\end{array}\right] \\
& W_{2}=\left[\begin{array}{cccc}
w_{n+1,1} & w_{n+1,2} & \cdots & w_{n+1, m} \\
w_{n+2,1} & w_{n+2,2} & \cdots & w_{n+2, m} \\
\vdots & \vdots & \vdots & \vdots \\
w_{n+k, 1} & w_{n+k, 2} & \cdots & w_{n+k, m}
\end{array}\right]^{T}
\end{aligned}
$$

Particle (2) $=\left[Z_{1}, Z_{2}\right]$

$$
Z_{1}=\left[\begin{array}{cccc}
z_{1,1} & z_{1,1} & \cdots & z_{1, k} \\
z_{2,1} & z_{2,1} & \cdots & z_{2, k} \\
\vdots & \vdots & \vdots & \vdots \\
z_{m, 1} & z_{m, 1} & \cdots & z_{m, k}
\end{array}\right]
$$




$$
Z_{2}=\left[\begin{array}{cccc}
Z_{1, k+1} & Z_{1, k+2} & \cdots & Z_{1, k+g} \\
Z_{2, k+1} & Z_{2, k+2} & \cdots & Z_{2, k+g} \\
\vdots & \vdots & \vdots & \vdots \\
Z_{m, k+1} & Z_{m, k+2} & \cdots & Z_{m, k+g}
\end{array}\right]
$$

Where $n$ is the dimension of input layer variables, $m$ is the number of hidden layer neurons, $k$ is the dimension of output layers, $g$ is the population size.

\subsection{Comparison Between Improved Network and Mathematical Precision Values}

To illustrate the proposed methodology, two numerical example were validated in this section.

Example 1

Consider a non-linear function with 5 uniform random variables. The function is:

$$
f(x)=-\sum_{i=1}^{4} c_{i} \exp \left[-\sum_{j=1}^{5} a_{i j}\left(x_{j}-p_{i j}\right)^{2}\right]
$$

$c_{i}=\left[\begin{array}{llll}1 & 1.2 & 3 & 3.2\end{array}\right], \quad x_{i} \in[0,1], i=1,2 \ldots 5$.

$$
\left[p_{i j}\right]_{j=1, \ldots, 5}=\left[\begin{array}{lllll}
0.1312 & 0.1696 & 0.5569 & 0.0124 & 0.8283 \\
0.2329 & 0.4139 & 0.8037 & 0.3736 & 0.1004 \\
0.2348 & 0.1451 & 0.3522 & 0.2883 & 0.3047 \\
0.4047 & 0.8828 & 0.8732 & 0.5743 & 0.1091
\end{array}\right]\left[a_{i j}\right]=\left[\begin{array}{ccccc}
1 & 0 & 0 & 1 & 0 \\
0 & 1 & 1 & 0 & 0 \\
0 & 0 & 1 & 0 & 0 \\
0 & 0 & 0 & 1 & 0 \\
0 & 0 & 0 & 0 & 1
\end{array}\right]
$$

Example 2

Consider a non-linear function with 5 uniform random variables. The function is:

$$
\begin{aligned}
& f(x)=\sum_{i=1}^{i=5} \sum_{j=1}^{j=5} a_{i j}\left(x_{i}^{2}+x_{i}+1\right)\left(x_{j}^{2}+x_{j}+1\right) \\
& x_{i} \in[-1,1], \quad x_{j} \in[-1,1] \\
& {\left[a_{i j}\right]=\left[\begin{array}{lllll}
1 & 0 & 0 & 1 & 0 \\
0 & 1 & 1 & 0 & 0 \\
0 & 0 & 1 & 0 & 0 \\
0 & 0 & 0 & 1 & 0 \\
0 & 0 & 0 & 0 & 1
\end{array}\right]}
\end{aligned}
$$

Tables $\mathbf{3}$ and $\mathbf{4}$ are the comparisons of the results in four aspects from the mean, the standard deviation, the number of samples, the maximum error and MAPE about Monte Carlo, neural network and improved neural network. The Monte Carlo results are the exact solutions that obtained from the Eq. (18) and Eq. (19) separately and abbreviated to MC.

The improved neural network is the proposed approximate model. The comparisons of the results of variable $x_{3}$ in improved network, general network and original equation are showed in Fig. (5) respectively. It is noted that the results obtained from the approximate model have higher precision by only uses 30 samples, the relative errors of the mean and the standard deviation are both lower than 3\%, MAPE less than 1\%. Furthermore, generating the approximate models of two examples required only 30 sample points. 
Table 3. Analysis results of neural networks for example 1.

\begin{tabular}{|c|c|c|c|c|c|}
\hline Statistic & Samples number & Mean & Standard deviation & Maximum error & MAPE(\%) \\
\hline MC & $10^{6}$ & -7.203 & 0.3774 & $/$ & $/$ \\
\hline improved neural network & 30 & -7.156 & 0.388 & 0.0008 & 0.612 \\
\hline relative error(\%) & $/$ & 0.652 & 2.81 & $/$ & $/$ \\
\hline network & 30 & -7.659 & 0.401 & 0.1362 & 9.3939 \\
\hline relative error(\%) & $/$ & 6.331 & 6.25 & $/$ & $/$ \\
\hline
\end{tabular}

Table 4. Analysis results of neural networks for example 2.

\begin{tabular}{|c|c|c|c|c|c|}
\hline Statistic & Samples number & Mean & Standard deviation & Maximum error & MAPE(\%) \\
\hline improved neural network & $10^{6}$ & 14.5574 & 6.5042 & $/$ \\
\hline MNN & 30 & 14.5573 & 6.5041 & 0.0022 \\
\hline relative error(\%) & $/$ & 0.0007 & 0.0015 & 0.342 \\
\hline ANN & 30 & 14.9856 & 6.4102 & $/$ \\
\hline network & $/$ & 2.9415 & 1.4452 & 4.312 & $/$ \\
\hline
\end{tabular}

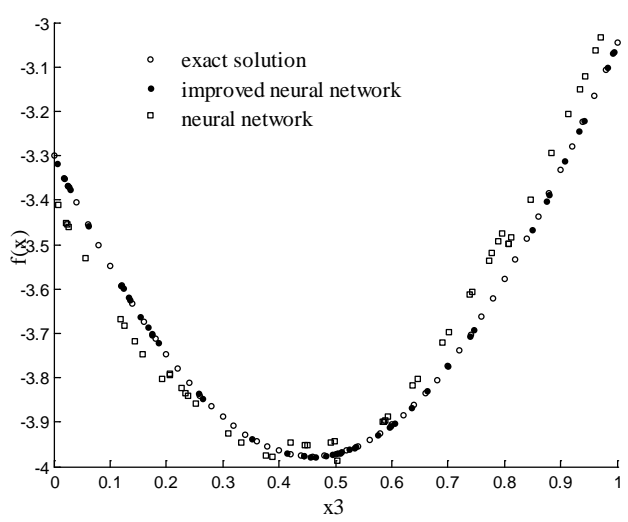

Fig. (5). Comparison of results.

\section{CALCULATION OF STRUCTURE SENSITIVITY COEFFICIENTS}

The sensitivity of each parameter is determined by the combination of the predicted results of neural network and Monte Carlo while considering the sampling of relativity according to probability density function. Considering the impact of each random variable on structure response, correlation coefficients are used to measure the correlation between random variables and structure responses. The coefficients of variation are brought in to eliminate the effect of the random variable dimensions and measurement scales. The equations are given by:

$$
\begin{aligned}
& R_{r}=\frac{\sum_{i=1}^{n}\left(x_{i}-\bar{x}\right)\left(f\left(x_{i}\right)-\overline{f\left(x_{i}\right)}\right)}{\sqrt{\sum_{i=1}^{n}\left(x_{i}-\bar{x}\right)^{2}\left(f\left(x_{i}\right)-\overline{f\left(x_{i}\right)}\right)^{2}}} \\
& S_{r}=\frac{R_{r}}{\gamma_{r}} / \sqrt{\left(\sum_{r=1}^{u} \frac{R_{r}}{\gamma_{r}}\right)^{2}}
\end{aligned}
$$

Where $R_{r}$ is the Pearson correlation coefficients, $x_{i}$ is random variable, $n$ is Monte Carlo sampling times, $f\left(x_{i}\right)$ is the network output according to $x_{i}, \bar{x}$ is the mean value of $x_{i}, \overline{f\left(x_{i}\right)}$ is the mean value of $f\left(x_{i}\right) ; \gamma_{r}$ is the coefficient of variation according to Monte Carlo random samples, $u$ is a number represented the random variables total dimensions, $S_{r}$ is the sensitivity value of random variables, the absolute value of coefficient reflects the influence of random 
variables on structure response, positive and negative reflect the degree of correlation between random variables and structure response.

\section{THE MOMENT-ROTATION MODEL OF EXTENDED END PLATE CONNECTION}

The finite element model of the extend end plate connection which has been verified in section 2.2 is used as the meta-model to construct the approximate model. Each simulation of extended end plate connection needs about ten minutes by finite element software. At least 100000 simulations to be employed for the sensitivity coefficients which are far beyond the computing power 50 finite element simulations are used to construct the approximate model and 100000 Monte Carlo samples are employed to get more accurate results by the proposed method. Eight different input parameters were used to model the moment-rotation response, these included beam height, beam width, the thickness of beam web, the thickness of beam flange, the thickness of plate, the distance between the middle bolts and the vertical axis of beam, the distance between the upper bolts and the vertical axis of beam and the distance between bolts and the vertical axis of beam web. The relative error between the results of the simulations and the approximate models is shown in Fig. (6).

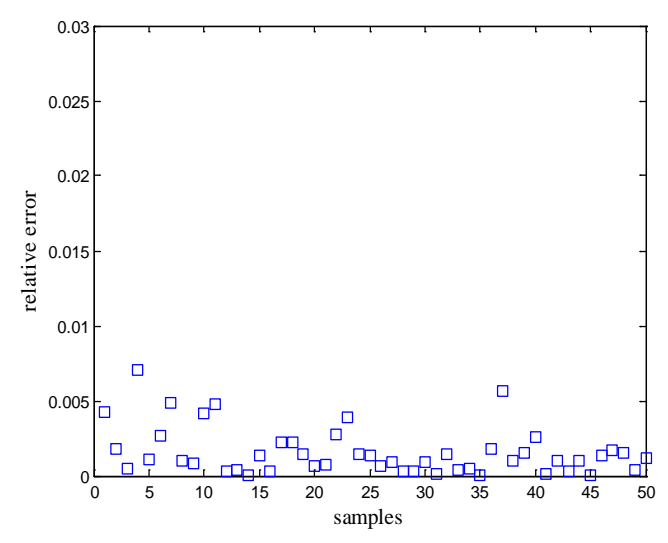

Fig. (6). Relative error.

Table 5 indicates the sensitivities $R_{r}$ and $R_{i}$ which conclude by considering relevant parameters and without considering relevant parameters. The sensitivity coefficient of beam width is -0.1544 without considering the correlation of the parameters. From the numerical point of view, the relationship between beam width and stiffness is not obvious. However, the actual situation is that the beam width is an important factor to the overall stiffness of the joint. In the process of the structure section size changed, the relation between the beam height and the width are correspondence, with the increasing of the beam height, the beam width is also appropriate increasing. By the way of considering the correlation between parameters, the sensitivity coefficient becomes -0.5257 , that shows the beam width has a great influence on the stiffness of the joint and this sensitivity agrees with the actual facts.

Table 5. Correlation coefficients of joint.

\begin{tabular}{|c|c|c|c|c|c|c|c|c|}
\hline \multirow{2}{*}{ Rotation } & \multicolumn{4}{|c|}{ BEAM } & \multicolumn{2}{c|}{ PLATE } & \multicolumn{3}{c|}{ BOLT } \\
\cline { 2 - 9 } & $\boldsymbol{h}$ & $\boldsymbol{b}$ & $\boldsymbol{t}$ & $\boldsymbol{t}_{\boldsymbol{w}}$ & $\boldsymbol{t}_{\boldsymbol{h}}$ & $\boldsymbol{h}_{\text {mid }}$ & $\boldsymbol{h}_{\boldsymbol{u}}$ & $\boldsymbol{V}$ \\
\hline$R_{r(i)}$ & -0.5925 & -0.5257 & -0.0004 & -0.0093 & -0.0508 & -0.2962 & 0.7316 & 0.1168 \\
\hline$R_{i(i)}$ & -0.4812 & -0.1544 & -0.0007 & -0.0653 & -0.2458 & -0.2956 & 0.7522 & 0.1196 \\
\hline
\end{tabular}

The moment-rotation model is composed by the parameters of the extended end plate connection, the sensitivity coefficients and the elastic modulus. The rotation at the left side of the equation is dimensionless. In order to make the dimensions same at both ends of the equation, the dimension of the parameters about the extended end plate connection should be $L^{-3}$ for the dimension of the moment is $M L^{2} S^{2}$ and the dimension of the elastic modulus is $M L^{-1} S^{-2}$, so the sensitivity coefficients should be transformed to standard coefficients and the transform process is expressed as Eq. (22).

The standard values of parameters sensitivity about extended end plate connection and the first order partial differential values $C_{i}$ between the parameters and the rotation are shown in the Table $\mathbf{6}$. The model is given by Eq. (23). Dimensions are same at both ends of the equation, improving the dimensions ranging from both ends by data fitting. 
Table 6. Integrated data.

\begin{tabular}{|c|c|c|c|c|c|c|c|c|}
\hline & \multicolumn{9}{|c|}{ BEAM } & PLATE & \multicolumn{3}{c|}{ BOLT } \\
\cline { 2 - 9 } & $\boldsymbol{h}$ & $\boldsymbol{b}$ & $\boldsymbol{t}$ & $\boldsymbol{t}_{\boldsymbol{w}}$ & $\boldsymbol{t}_{\boldsymbol{h}}$ & $\boldsymbol{h}_{\text {mid }}$ & $\boldsymbol{h}_{\boldsymbol{u}}$ & $\boldsymbol{V}$ \\
\hline$R_{s(i)}$ & -2.837 & -2.517 & -0.002 & -0.045 & -0.243 & -1.418 & 3.503 & 0.559 \\
\hline$C_{i}$ & -.0032 & -.0056 & -.000065 & -.0021 & -.0082 & -.0048 & .0059 & .0038 \\
\hline$\beta_{i}$ & 61.0651 & 60.2009 & 64.12 & 58.9512 & 60.6402 & 61.0831 & 57.7986 & 55.2033 \\
\hline$\beta_{a}$ & \multicolumn{9}{|c|}{59.88} \\
\hline
\end{tabular}

$$
\begin{gathered}
R_{s(i)}=\frac{3 R_{r(i)}}{\sum R_{r}} \\
\theta_{r}=\frac{\beta h_{u p}^{3.503} V^{0.559} M}{E h^{2.837} b^{2.517} t^{0.002} t_{w}^{0.045} t_{h}^{0.243} h_{\text {mid }}^{1.418}} \\
\frac{\partial \theta_{r}}{\partial h}=\frac{\partial}{\partial h}\left(\frac{\beta_{h} h_{u p}^{3.503} V^{0.559} M}{E_{0} h^{2.837} b^{2.517} t^{0.002} t_{w}^{0.045} t_{h}^{0.243} h_{\text {mid }}^{1.418}}\right)=C_{h}
\end{gathered}
$$

Where $\theta_{r}$ is the rotation between beam and column, $M$ is the moment of beam end, $h$ is the beam height, $b$ is the beam width, $t$ is the thickness of beam web, $t_{w}$ is the thickness of beam flange, $t_{h}$ is the thickness of plate, $h_{\text {mid }}$ is the distance between the middle bolts and the vertical axis of beam, $h_{u p}$ is the distance between the upper bolts and the vertical axis of beam, $V$ is the distance between bolts and the vertical axis of beam web, $\beta$ is the mean correction coefficient about $\beta_{i}$. For example, $\beta_{h}$ can be obtained by the Eq. (24).

\section{CONCLUSION}

A method of the moment rotation model of semi-rigid connection has been proposed in this paper. The main algorithm includes Monte Carlo sampling with the correlation of the parameters, the approximate model and the method of stochastic sensitivity, moreover, the correlation of the parameters as an essential component affects the whole structure systems.

A key feature of the approximate model is the hybrid neural network procedure that efficiently deals with simulation models and progressively trains a set of metamodels. As part of this study, the effectiveness of the proposed method, which does not require huge finite element simulations, was optimized by the improved chaos-particle swarm provided a satisfactory approach for highly non-linear functions. Two high dimensional equations have been used to be examined and agreement appears much closer between these results which were calculated by approximate model and the Monte Carlo results. Furthermore, the approximate model has the high efficiency for only dozens of samples.

Finite element analysis of the extend end plate connection is carried out by ANSYS software, the results have been compared with the tests results and reported a high degree of accuracy, the maximum relative error is less than $1 \%$. In addition, these results of approximate models have been used for sensitivity analysis in moment-rotation model. The calculations of the sensitivity coefficients considering parameters correlation are more consistent with the actual. The relative moment-rotation model was established based on the sensitivity coefficients of parameters and stochastic analysis. Elastic modulus was brought in the regression equation of moment rotation for the point of mechanics to make both ends of the equation equivalent from the dimension, so that the regression equation is more than the numerical fitting moment rotation curve. Furthermore, the accuracy and efficiency make the proposed methods of the approximate model and the method of stochastic sensitivity suitable to be implemented in other types of the steel connections.

\section{CONFLICT OF INTEREST}

The authors confirm that this article content has no conflict of interest. 


\section{ACKNOWLEDGEMENTS}

This work is supported by National Natural Science Foundation of China under the grant number 51378009, 51378219 .

\section{REFERENCES}

[1] F.H. Wu, Semi-rigid Connection in Steel Frames., College of Engineering, Purdue University: Purdue, 1989.

[2] Y.L. Yee, and R.E. Yee, "Moment-rotation curves for bolted connections", J. Struct. Eng., vol. 112, no. 3, pp. 615-635, 1993. [http://dx.doi.org/10.1061/(ASCE)0733-9445(1986)112:3(615)]

[3] F.H. Wu, and W.F. Chen, "A design model for semi-rigid connections", Eng. Struct., vol. 12, no. 2, pp. 88-97, 1990. [http://dx.doi.org/10.1016/0141-0296(90)90013-I]

[4] J.Y. Liew, D.W. White, and W.F. Chen, "Second-order refined plastic hinge analysis for frame design: Part 1", J. Struct. Eng., vol. 119, no. 11, pp. 3196-3216, 1993. [http://dx.doi.org/10.1061/(ASCE)0733-9445(1993)119:11(3196)]

[5] S.E. Kim, and W.F. Chen, "Practical advanced analysis for braced steel frames design", J. Struct. Eng., vol. 122, no. 11, pp. 1266-1274, 1996. [http://dx.doi.org/10.1061/(ASCE)0733-9445(1996)122:11(1266)]

[6] S.L. Chan, and C.Y. Yau, "Inelastic and stability analysis of flexibly connected steel frames by springs-in-series model", J. Struct. Eng., vol. 120, no. 10, pp. 2803-2819, 1994. [http://dx.doi.org/10.1061/(ASCE)0733-9445(1994)120:10(2803)]

[7] S. Hu, "An efficient computational method for semi-rigid steel frame with elements of internal plastic hinge", J. Inf. Comput. Sci., vol. 11, no. 3, pp. 833-844, 2014.

[http://dx.doi.org/10.12733/jics20102976]

[8] A. Saritas, A. Koseoglu, and H.F. Ozel, "Modeling of the nonlinear behavior of steel framed structures with semi-rigid connections", In: Eighth International Conference on Advances in Steel Strictures, Lisbon: Portugal, 2015, pp. 1-18.

[9] C. Xu, and G.Z. Gertner, "Uncertainty and sensitivity analysis for models with correlated parameters", Reliab. Eng. Syst. Saf., vol. 93, no. 10, pp. 1563-1573, 2008.

[http://dx.doi.org/10.1016/j.ress.2007.06.003]

[10] D. Xian, and W. Zhan, "Stochastic sensitivity analysis method based on correlation parameters and hybrid neural network", J. Build. Struct., vol. 36, no. 4, pp. 149-157, 2015. [in Chinese].

[11] D. Xian, W. Zhan, and J. Pan, "Stochastic sensitivity analysis for models with parameter correlation and model approximation method", J. Inf. Comput. Sci., vol. 12, no. 2, pp. 657-672, 2015.

[http://dx.doi.org/10.12733/jics20105305]

[12] F.W. Wang, "Optimization and sensitivity analysis of space frames allowing for large deflection", Eng. Struct., vol. 10, pp. 1395-1406, 2006.

[13] A.M. Girão Coelho, F.S. Bijlaard, and L. Simões da Silva, "Experimental assessment of the ductility of extended end plate connections", Eng. Struct., vol. 26, no. 9, pp. 1185-1206, 2004. [http://dx.doi.org/10.1016/j.engstruct.2000.09.001]

[14] J. Pan, Analytical method on semi-rigid joint of framework based on correlations., Shantou University: China, 2009. [in Chinese]

[15] A. Der Kiureghian, and P.L. Liu, "Structural reliability under incomplete probability information", J. Eng. Mech., vol. 112, no. 1, pp. 85-104, 1986. [http://dx.doi.org/10.1061/(ASCE)0733-9399(1986)112:1(85)]

[16] J. Pan, and W. Zhan, "Semirigid analysis of extended end plate connections based on correlation and sensitivity", J. Shanghai Jiaotong Univ. (Sci.), vol. 17, no. 5, pp. 598-604, 2012. [http://dx.doi.org/10.1007/s12204-012-1331-8]

[17] K.S. Al-Jabri, S.M. Al-Alawi, A.H. Al-Saidy, and A.S. Alnuaimi, "An artificial neural network model for predicting the behaviour of semirigid joints in fire", Adv. Steel Constr., vol. 5, no. 4, pp. 452-464, 2009.

[18] K.M. Hornik, M. Stinchcombe, and H. White, "Multilayer feedforward network s are universal approximators", Neural Netw., vol. 2, no. 2, pp. 359-366, 1989.

[http://dx.doi.org/10.1016/0893-6080(89)90020-8]

[19] M.R. Far, M.Z. Jumaat, and R.T. Vahid, "Nonlinear analysis of load-deflection testing of reinforced one-way slab strengthened by carbon fiber reinforced polymer (CFRP) and using artificial neural network (ANN) for prediction", Int. J. Phys. Sci., vol. 6, no. 13, pp. 3054-3061, 2011

[20] Guang-zhi Yin, and Ming-hui Li, "Model of coal gas permeability prediction based on improved BP neural network", J. China Coal Soc., vol. 36, no. 7, pp. 1180-1184, 2013. [in Chinese].

[21] H. Han, J. Qiao, and Y. Bo, "On structure design for RBF neural network based on information strength", Acta Automatica Sinica, vol. 38, no. 7, pp. 1083-1090, 2012. [in Chinese]. [http://dx.doi.org/10.3724/SP.J.1004.2012.01083] 
[22] G. Fei, and H. Tong, "Parameter estimation for system based on particle swarm optimization", Acta. Phys., vol. 55, no. 2, pp. 577-582, 2006. [in Chinese].

[23] X. Xu, K. Zheng, and L. Dan, "New chaos-particle swarm optimization algorithm", J. Commun., vol. 33, no. 1, pp. 24-29, 2012. [in Chinese].

[24] Dejian Lai, Guanrong Chen, and Martin Hasler, "Distribution of the lyapunov exponent of the chaotic skew tent map", Int. J. Bifurcat Chaos., vol. 9, no. 9, pp. 2059-2067, 2011.

C Dong and Wang; Licensee Bentham Open

This is an open access article licensed under the terms of the Creative Commons Attribution-Non-Commercial 4.0 International Public License (CC BY-NC 4.0) (https://creativecommons.org/licenses/by-nc/4.0/legalcode), which permits unrestricted, non-commercial use, distribution and reproduction in any medium, provided the work is properly cited. 